\title{
Data assimilation for a visco-elastic Earth deformation model
}

Reyko Schachtschneider ${ }^{1}$, Jan Saynisch-Wagner ${ }^{1}$, Meike Bagge $^{1}$, Volker Klemann ${ }^{1}$, Maik Thomas ${ }^{1,2}$

1: GFZ German Research Centre for Geosciences, Potsdam, Germany

2: Free University, Berlin, Germany

EGU, 2020-05-05 


\section{The model VILMA}



- Model for Earth's visco-elastic deformation due to glaciation / deglaciation

- Capable of handling 3D viscosity distributions

- Calculates deformation with spectral FE method in time domain

- Solves sea-level equation, i.e. relative sea level (RSL)

Aim of this study:

Parameter estimation of viscosity distribution in the mantle

\section{G F Z}




\section{Data assimilation setup}

- Sandbox setup $\rightarrow$ use pseudo sea-level data of reference run $m_{0}$ as observations

- Assimilate sea-level data in order to gain information about viscosity structure

- Assimilation starts shortly before last glacial maximum $\left(t_{0}=-26.5 \mathrm{kyr}\right)$

- Observations available every $1 \mathrm{kyr}$

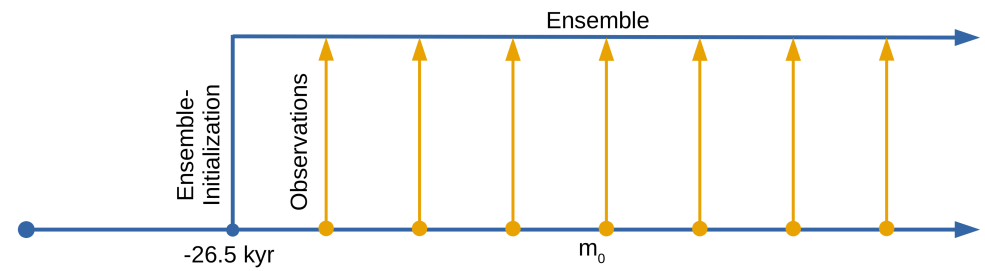

\section{G F Z}




\section{Results I - RMS error \& likelihood}
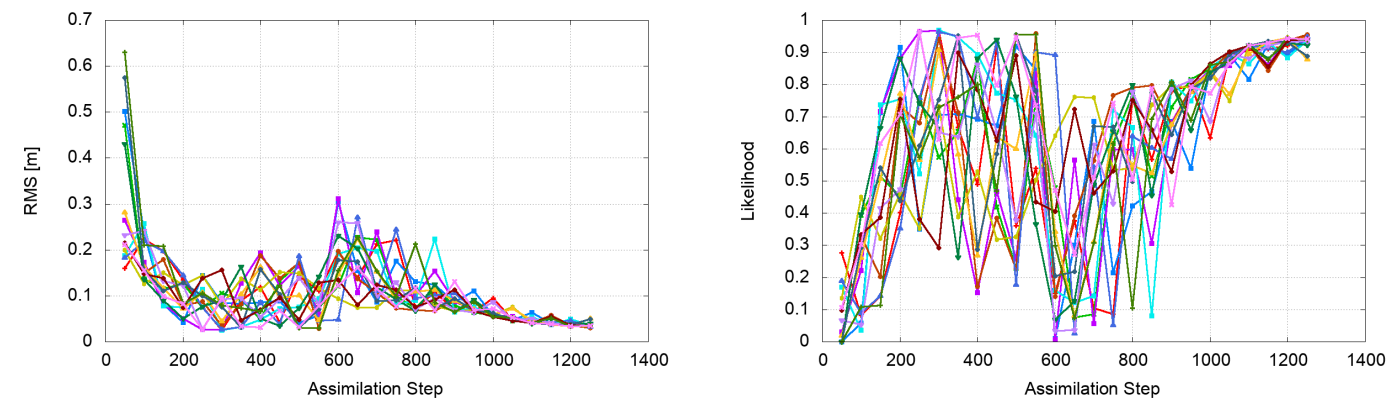

RMS of differences between observations and model state (left). Likelihood of observations (right). Each color represents one ensemble member. The observations are relative sea levels (RSL).

\section{G F Z}




\section{Results II - Viscosities development}
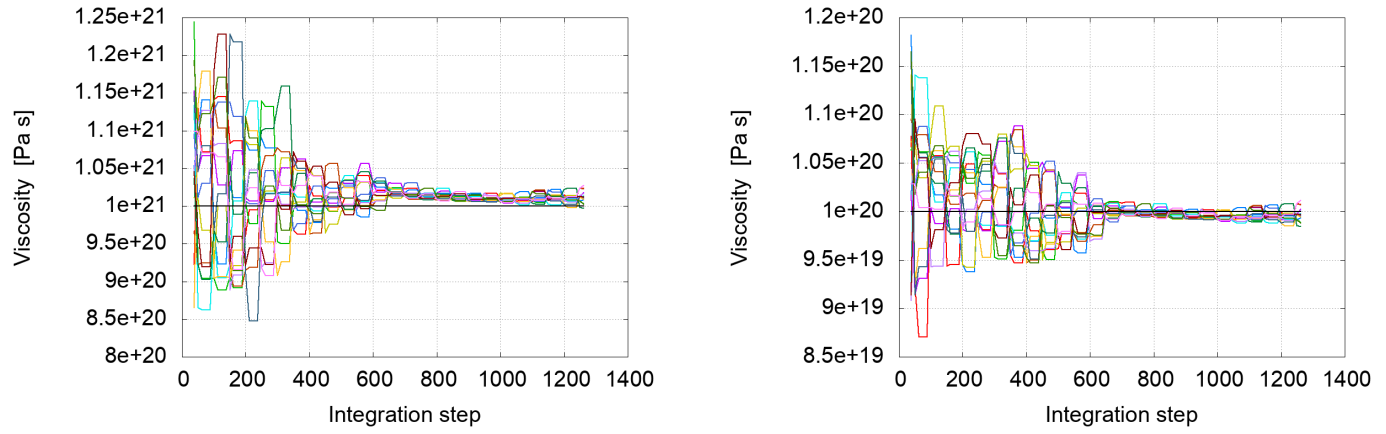

Development of viscosity values for lower mantle (left) and upper mantle (right). The horzontal black line represents the viscosity value of the reference run from which the observations were taken. Data assimilation was performed every 50 steps. In the mantle regions, the ensemble converges towards the reference run.

\section{G F Z}




\section{Summary}

- Successfully added data assimilation algorithm to VILMA

- Recovered a given viscosity structure from synthetic relative sea level data

- Model converged well in mantle regions

reyko.schachtschneider@gfz-potsdam.de 